\title{
Personalized medicine in multiple sclerosis: hope or reality?
}

Tobias Derfuss ${ }^{1,2}$

\begin{abstract}
Personalized treatment is highly desirable in multiple sclerosis because it is an immensely heterogeneous disease. This heterogeneity is seen in both the disease course and the treatment responses. Currently, a combination of clinical features and imaging parameters in magnetic resonance imaging is used to classify active and non-active patients and treatment responders and non-responders. Although this classification works on a group level, individual patients often behave differently from the group. Therefore additional biomarkers are needed to provide better indicators for prognosis and treatment response. Basic and clinical research have discovered different promising targets. It is now essential to verify the utility and accuracy of these markers in large, prospectively sampled patient cohorts.
\end{abstract}

Keywords: biomarker, multiple sclerosis, personalized medicine

\section{Review}

Background

Multiple sclerosis (MS) is a chronic autoimmune disease of the central nervous system. Because it hits patients early during life it has a major impact on a large part of their lives, and imposes a considerable economic burden. Current treatments for MS mainly target inflammatory processes, and there has been scant progress in treatments that enhance neuronal or glial regeneration. Therefore the current treatment strategy is to start treatment early to prevent neurodegeneration from the beginning. However, different problems arise when patients are treated after the first sign of the disease. First, does the patient need treatment at all, because he or she may have a benign disease

\footnotetext{
Correspondence: TDerfuss@uhbs.ch

'Department of Neurology, University Hospital Basel, Petersgraben 4, 4031

Basel, Switzerland

Full list of author information is available at the end of the article
}

course? Second, to which of the current baseline therapies will the patient respond best? Third, does the patient need an induction therapy? Fourth, is the patient at an increased risk for serious side effects?

Data from clinical trials provide information about efficacy and safety on a group level. However, it is obvious that treatment decisions in clinical practice must be made on an individual basis. This requires a personalized medicine approach. Biomarkers that could predict disease course, treatment response, and risk of side effects would be highly appreciated. Despite extensive research over the last years, few biomarkers have made their way into clinical practice. This mini review aims to summarize the state of current biomarker development in MS and promising new approaches.

\section{Predicting disease activity in multiple sclerosis}

MS is a highly heterogeneous disease. This is probably not only true for the etiology, pathological features and autoantigenic targets but also for the disease course and response to treatment. Data from natural history cohorts show a broad spectrum of disease severities. The disease course is benign in $10 \%$ to $15 \%$ of patients and they do not need an assistive device for walking even after 20 years of MS [1]. On the other end of the spectrum, there are fulminant courses of MS that lead to severe disability within a few years. This heterogeneity of disease severity has obvious consequences for treatment decisions. In patients with a more aggressive disease, there is a need for early and aggressive treatment. Although this aggressive treatment poses some risks, these risks would be accepted by the patient and treating physician knowing that the benefits in this specific patient outweigh the potential risks.

Can we predict the disease course? There seem to be some clinical indicators that point to a worse disease course, such as high relapse rate during the first two to three years, bad recovery from relapses, and motor symptoms early during disease [2-4] (although these predictors have been disputed by others $[5,6])$. In addition, paraclinical tests such as high lesion load in magnetic resonance 
Table 1 Summary of established and potential biomarkers for diagnosis, prognosis, and treatment of multiple sclerosis.

\begin{tabular}{lccc}
\hline Established biomarkers & Diagnosis & Prognosis & Treatment response/side effects \\
\hline Cerebral spinal fluid-specific oligoclonal bands & + & $(+)$ & - \\
Intrathecal immunoglobulin production & + & $(+)$ & - \\
Intrathecal anti-viral immunoglobulin production & + & - & - \\
Magnetic resonance imaging & + & $(+)$ & $(+)$ \\
Neutralizing antibodies against beta-interferon & - & - & $(+)$ \\
Neutralizing antibodies against natalizumab & - & - & + \\
Antibodies against JC virus & - & + & $(+)$ \\
Aquaporin 4 antibodies & + & - & $(+)$ \\
Potential biomarkers & - & - & $(+)$ \\
CD56 bright natural killer cells & - & - & - \\
Cytokines/chemokines & $(+)$ & $(+)$ & - \\
Myelin oligodendrocyte glycoprotein antibodies & - & - & $(+)$ \\
Intrathecal/oligoclonal immunoglobulin M production & - & $(+)$ & $(+)$ \\
Transcriptomics & & - & \\
Genetics & & - & \\
\hline
\end{tabular}

+ valid biomarker that is used in clinical practice; $(+)$ there is experimental and clinical evidence for this biomarker, but the need for more clinical data; - there is no clear evidence.

imaging (MRI), lipid-specific IgM oligoclonal bands and certain electrophysiological parameters hint at a more active disease (Table 1) [7-9]. However, while all these factors work fine on a group level, their predictive power in individual patients is low. Nevertheless, MRI is extensively used in clinical practice as a surrogate marker for the burden and activity of disease [10]. It can be expected that compound MRI measures that combine classical sequences like T2 and contrast enhanced T1 with more sophisticated measures like diffusion tensor imaging, double inversion recovery and magnetization transfer ratio will enhance the predictive power of MRI [11]. These techniques will allow for the detection of grey matter lesions that occur early during the disease course and that seem to correlate better with disability than white matter lesions that are detected with the standard T2-weighed imaging [12].

How to pick the right medication for the right patient?

To date, choosing the right first-line therapy is based on guessing rather than knowing. On the one hand, one has to consider the activeness and severity of the disease when choosing a therapy. On the other hand, one has to take into account that no treatment has a responder rate of $100 \%$. If a patient is placed on a treatment that does not work perfectly, this patient will lose precious time and will still have the risks of the treatment. In addition, society will have costs without benefit. Therefore there is an immense need to establish biomarkers that can predict treatment responses. So far, the response to a treatment is judged by counting clinical relapses, disability progression, and new lesions in MRI after one year of treatment [13]. Established biomarkers that are correlated with treatment responses include neutralizing antibodies against IFNs and natalizumab (Table 1) $[14,15]$. Genetic markers have not proven to be of use in predicting treatment response, so far. Recent studies on transcriptional profiles (both mRNA as well as miRNA) might reveal IFN response markers but this has to be reproduced in further longitudinal patient cohorts [16].

Another class of biomarkers is defined by the detection of pathologic immune responses, primarily antibody responses, against putative autoantigens [17]. These have been established as biomarkers in other neuroimmunological diseases such as myasthenia (antibodies against acetylcholine receptor) and paraneoplastic disorders (anti-Hu, anti-Yo and so on). In demyelinating diseases of the central nervous system, aquaporin 4 (AQP4) and myelin oligodendrocyte glycoprotein (MOG) have emerged as interesting antibody targets. Antibodies against AQP4 are associated with the clinical spectrum of neuromyelitis optica [18]. Because neuromyelitis optica seems to have a more aggressive course than general MS and seems to respond better to classical immunosuppression than to immunomodulation, detection of AQP4 antibodies helps to classify patients and aids in treatment decisions. Antibody responses against MOG are mainly found in pediatric demyelinating diseases like acute disseminated encephalomyelitis and pediatric MS [19]. MOG antibodies might help in differentiating between viral and autoimmune encephalitis [20]. Monophasic acute disseminated encephalomyelitis and pediatric MS might also be separated by MOG antibodies because these antibodies tend to persist longer in pediatric MS [19]. However, more longitudinal data are needed to corroborate this finding. If it is true, persisting anti-MOG antibodies would aid in guiding 
prophylactic treatment regimens. The role of MOG antibodies in adult MS is still speculative. More research is needed to clarify if MOG antibodies can be used for prognosis or classification of adult MS patients. A very recent discovery is the increased humoral immune response against KIR4.1 that was found in different cohorts of patients with MS compared with different control cohorts [21]. KIR4.1 is a rectifying potassium channel expressed by astrocytes and oligodendrocytes. Injection of KIR4.1 specific IgG (derived from MS sera) into the cisterna magna of mice caused pathological changes like complement deposition and loss of KIR4.1 antigen. As with MOG, it remains to be seen if the immune response against KIR4.1 will be useful as a biomarker for diagnosis, prognosis or treatment responses.

Another novel biomarker that may predict treatment responses early during therapy was discovered during clinical development of daclizumab, a monoclonal anti-CD25 antibody. It was shown that blocking the high-affinity IL-2 receptor (CD25) by daclizumab led to the expansion of a subtype of NK cells that show a high expression of CD56. This cell type seems to have immunoregulatory functions [22]. Expansion of CD56bright NK T-cells correlated with decreased MRI activity during daclizumab therapy in a phase 2 trial and may therefore indicate a patient population that preferentially responds to this treatment [23]. Despite these promising new avenues of research, we are currently left with clinical markers of treatment responses.

\section{Can we predict the risk of serious side effects?}

When using immunosuppressive or immunomodulatory treatment, we are often confronted with serious side effects such as increased risk for infections. These risks often increase with the effectiveness of the treatments. A prominent example is the treatment with natalizumab, a monoclonal antibody against an integrin that inhibits migration of lymphocytes into the brain. This treatment shows an impressive reduction of relapses and disease activity in MRI [24]. The major drawback of this treatment is, however, the increased risk for a progressive multifocal leukoencephalopathy (PML) [25]. A careful review of PML cases in a post-marketing safety program revealed that a longer duration of therapy and previous immunosuppressive treatment are correlated with an increased risk of PML. As far as we know, PML is a reactivation of a preexisting latent infection with JC virus. A specific ELISA for the causative JC virus was developed that indicates if a patient harbors latent JC virus [26]. Using these three parameters (treatment duration, previous immunosuppressive treatment, JC virus antibody status), a risk stratification algorithm has been established that can be used in clinical practice to counsel patients that are on current natalizumab treatment or that are suited to go on the treatment [25].
Another biomarker that could predict autoimmune side effects of a treatment with alemtuzumab (monoclonal antibody against CD52 that depletes lymphocytes and monocytes) has been identified in early clinical studies. A surprising finding was made during the early clinical development of alemtuzumab: the occurrence of autoimmune thrombocytopenia and thyroid disease [27]. The pretreatment levels of IL-21 in serum correlated with the later development of autoimmune reactions [28]. Obviously, more prospective data are needed to confirm the value of this test in clinical practice.

The genetic background of a patient could also be used as a personalized biomarker. With the advent of high-throughput genetic screening approaches, genetic data are available in high quality and at ever decreasing costs. Some health authorities already demand genetic testing for certain human leukocyte antigen (HLA) haplotypes to predict the risk of serious cutaneous adverse effects of carbamazepine treatment [29]. In MS, an increasing number of genetic polymorphisms have been correlated with the disease [30] but so far their power to aid in diagnosing MS is low [31]. A large number of genes (including GSTM, IL1B, PD-1, CCR5, OPN, IL4, HLA-DRB1*1501, CD24, ESR1, CD59, CNTF, CRYAB, IFN $\gamma, M E F V, A P O E, T G F B 1$ ) have been associated with certain MS phenotypes but these correlations were often controversial [32].

Research on the pharmacogenomics of MS is increasing but a useful biomarker for clinical practice has so far not emerged [33]. Nevertheless, a recent study analyzing the functional consequences of a TNF receptor 1 polymorphism linked to MS shed light on possible reasons why a TNF $\alpha$ blockade failed as therapy in MS when they have been effective for other autoimmune diseases [34,35]. Gregory and colleagues [35] showed that this polymorphism leads to a novel soluble TNF receptor that can block TNF $\alpha$, suggesting that a TNF $\alpha$ blockade in MS contributes to its pathogenesis rather than protecting from it. This study suggests that the functional analysis of genetic variants might help to predict autoimmune side effects related to specific immune pathways.

\section{Future directions and conclusions}

The treatment armamentarium of MS has increased tremendously over the last few years and more treatments are close to registration. Although a final cure for MS is still missing, MS will be manageable in most patients with these treatments. The most important challenge regarding these therapeutic interventions will be to tailor the therapy to the needs of the patients and the aggressiveness of the disease. This asks for the development of biomarkers, either clinical, genetic, imaging or immunological, that allow for a better stratification of patients. An important prerequisite of biomarker development is the availability 
of longitudinal patient cohorts that are followed up over years. These cohorts could provide prospectively collected clinical information as well as genetic, imaging and immunological data. Many biomarkers have been proposed in clinical research. To finally bring them to clinical practice needs academic and industry co-operation. This joint effort will bring us one step closer from the hope to the reality of personalized medicine in MS.

\section{Acknowledgements}

I'm grateful to Nicholas Sanderson for helpful comments.

\section{Abbreviations}

AQP4: aquaporin 4; ELISA: enzyme-linked immunosorbent assay; IFN: interferon; HLA: human leukocyte antigen; Ig: immunoglobulin; IL: interleukin; miRNA: microRNA; MOG: myelin oligodendrocyte glycoprotein; MRI: magnetic resonance imaging; MS: multiple sclerosis; NK: natural killer; PML: progressive multifocal leukoencephalopathy; TNF: tumor necrosis factor.

\section{Author details}

'Department of Neurology, University Hospital Basel, Petersgraben 4, 4031 Basel, Switzerland. '2Department of Biomedicine, University Hospital Basel, Hebelstrasse 20,4031 Basel, Switzerland.

\section{Authors' information}

TD is a clinical neurologist who specializes in neuroimmunology. He heads the outpatient and MS center at the Department of Neurology and is research professor at the Department of Biomedicine at the University in Basel. His research focuses on the discovery of new autoantigens, on biomarkers and on the analysis of the mode of action of disease-modifying treatments in MS. He is also involved in clinical trials for newly emerging therapies in MS.

\section{Competing interests}

TD serves on scientific advisory boards for Novartis, Merck Serono, Mitsubishi Pharma, Biogen Idec, Teva and Bayer Schering Pharma; has received funding for travel and/or speaker honoraria from Biogen Idec, Novartis, Merck Serono, and Bayer Schering Pharma; and receives research support from Biogen Idec, Novartis, Merck Serono, the European Union, and the Swiss MS Society.

Received: 25 July 2012 Accepted: 4 October 2012

Published: 4 October 2012

\section{References}

1. Skoog B, Runmarker B, Winblad S, Ekholm S, Andersen O: A representative cohort of patients with non-progressive multiple sclerosis at the age of normal life expectancy. Brain 2012, 135:900-911.

2. Runmarker $B$, Andersen $O$ : Prognostic factors in a multiple sclerosis incidence cohort with twenty-five years of follow-up. Brain 1993, 116(Pt 1):117-134.

3. Confavreux C, Vukusic S, Adeleine P: Early clinical predictors and progression of irreversible disability in multiple sclerosis: an amnesic process. Brain 2003, 126:770-782.

4. Weinshenker BG, Bass B, Rice GP, Noseworthy J, Carriere W, Baskerville J, Ebers GC: The natural history of multiple sclerosis: a geographically based study. 2. Predictive value of the early clinical course. Brain 1989, 112(Pt 6):1419-1428.

5. Kremenchutzky M, Rice GP, Baskerville J, Wingerchuk DM, Ebers GC: The natural history of multiple sclerosis: a geographically based study 9 : observations on the progressive phase of the disease. Brain 2006, 129:584-594

6. Scalfari A, Neuhaus A, Degenhardt A, Rice GP, Muraro PA, Daumer M, Ebers GC: The natural history of multiple sclerosis: a geographically based study 10: relapses and long-term disability. Brain 2010, 133:1914-1929.
7. Fisniku LK, Brex PA, Altmann DR, Miszkiel KA, Benton CE, Lanyon R, Thompson AJ, Miller DH: Disability and T2 MRI lesions: a 20-year followup of patients with relapse onset of multiple sclerosis. Brain 2008, 131:808-817.

8. Schlaeger R, D'Souza M, Schindler C, Grize L, Dellas S, Radue EW, Kappos L, Fuhr P: Prediction of long-term disability in multiple sclerosis. Mult Scler 2012, 18:31-38

9. Villar LM, Sadaba MC, Roldan E, Masjuan J, Gonzalez-Porque P, Villarrubia N, Espino M, Garcia-Trujillo JA, Bootello A, Alvarez-Cermeno JC: Intrathecal synthesis of oligoclonal IgM against myelin lipids predicts an aggressive disease course in MS. J Clin Invest 2005, 115:187-194.

10. Tintore M, Rovira A, Arrambide G, Mitjana R, Rio J, Auger C, Nos C, Edo MC, Castillo J, Horga A, Perez-Miralles F, Huerga E, Comabella M, Sastre-Garriga J, Montalban X: Brainstem lesions in clinically isolated syndromes. Neurology 2010, 75:1933-1938.

11. Bakshi R, Thompson AJ, Rocca MA, Pelletier D, Dousset V, Barkhof F, Inglese M, Guttmann CR, Horsfield MA, Filippi M: MRI in multiple sclerosis: current status and future prospects. Lancet Neurol 2008, 7:615-625.

12. Fisniku LK, Chard DT, Jackson JS, Anderson VM, Altmann DR, Miszkiel KA, Thompson AJ, Miller DH: Gray matter atrophy is related to long-term disability in multiple sclerosis. Ann Neurol 2008, 64:247-254.

13. Rio J, Comabella M, Montalban X: Predicting responders to therapies for multiple sclerosis. Nat Rev Neurol 2009, 5:553-560.

14. Polman $\mathrm{CH}$, Bertolotto A, Deisenhammer F, Giovannoni G, Hartung HP, Hemmer B, Killestein J, McFarland HF, Oger J, Pachner AR, Petkau J, Reder AT, Reingold SC, Schellekens H, Sørensen PS: Recommendations for clinical use of data on neutralising antibodies to interferon-beta therapy in multiple sclerosis. Lancet Neurol 2010, 9:740-750.

15. Deisenhammer F: Neutralizing antibodies to interferon-beta and other immunological treatments for multiple sclerosis: prevalence and impact on outcomes. CNS Drugs 2009, 23:379-396.

16. Comabella M, Lunemann JD, Rio J, Sanchez A, Lopez C, Julia E, Fernandez M, Nonell L, Camina-Tato M, Deisenhammer F, Caballero E, Tortola MT, Prinz M, Montalban X, Martin R: A type I interferon signature in monocytes is associated with poor response to interferon-beta in multiple sclerosis. Brain 2009, 132:3353-3365.

17. Derfuss T, Meinl E: Identifying autoantigens in demyelinating diseases: valuable clues to diagnosis and treatment? Curr Opin Neurol 2012 25:231-238.

18. Hinson SR, McKeon A, Lennon VA: Neurological autoimmunity targeting aquaporin-4. Neuroscience 2010, 168:1009-1018.

19. Probstel AK, Dornmair K, Bittner R, Sperl P, Jenne D, Magalhaes $S$, Villalobos A, Breithaupt C, Weissert R, Jacob U, Krumbholz M, Kuempfel T, Blaschek A, Stark W, Gärtner J, Pohl D, Rostasy K, Weber F, Forne I, Khademi M, Olsson T, Brilot F, Tantsis E, Dale RC, Wekerle H, Hohlfeld R, Banwell B, Bar-Or A, Meinl E, Derfuss T: Antibodies to MOG are transient in childhood acute disseminated encephalomyelitis. Neurology 2011, 77:580-588.

20. Lalive PH, Hausler MG, Maurey $H$, Mikaeloff $Y$, Tardieu M, Wiendl $H$, Schroeter M, Hartung HP, Kieseier BC, Menge T: Highly reactive anti-myelin oligodendrocyte glycoprotein antibodies differentiate demyelinating diseases from viral encephalitis in children. Mult Scler 2011, 17:297-302.

21. Srivastava R, Aslam M, Kalluri SR, Schirmer L, Buck D, Tackenberg B, Rothhammer V, Chan A, Gold R, Berthele A, Bennett JL, Korn T, Hemmer B: Potassium channel KIR4.1 as an immune target in multiple sclerosis. $N$ Engl J Med 2012, 367:115-123.

22. Bielekova B, Catalfamo M, Reichert-Scrivner S, Packer A, Cerna M, Waldmann TA, McFarland H, Henkart PA, Martin R: Regulatory CD56(bright) natural killer cells mediate immunomodulatory effects of IL-2Ralphatargeted therapy (daclizumab) in multiple sclerosis. Proc Natl Acad Sci USA 2006, 103:5941-5946.

23. Wynn D, Kaufman M, Montalban X, Vollmer T, Simon J, Elkins J, O'Neill G, Neyer L, Sheridan J, Wang C, Fong A, Rose JW, CHOICE investigators: Daclizumab in active relapsing multiple sclerosis (CHOICE study): a phase 2, randomised, double-blind, placebo-controlled, add-on trial with interferon beta. Lancet Neurol 2010, 9:381-390.

24. Polman $\mathrm{CH}, \mathrm{O}^{\prime}$ Connor PW, Havrdova E, Hutchinson M, Kappos L, Miller DH, Phillips JT, Lublin FD, Giovannoni G, Wajgt A, Toal M, Lynn F, Panzara MA, Sandrock AW, AFFIRM Investigators: A randomized, placebo-controlled trial of natalizumab for relapsing multiple sclerosis. N Engl J Med 2006, 354:899-910 
25. Bloomgren G, Richman S, Hotermans C, Subramanyam M, Goelz S, Natarajan A, Lee S, Plavina T, Scanlon JV, Sandrock A, Bozic C: Risk of natalizumab-associated progressive multifocal leukoencephalopathy. N Engl J Med 2012, 366:1870-1880.

26. Gorelik L, Lerner M, Bixler S, Crossman M, Schlain B, Simon K, Pace A, Cheung A, Chen LL, Berman M, Zein F, Wilson E, Yednock T, Sandrock A, Goelz SE, Subramanyam M: Anti-JC virus antibodies: implications for PML risk stratification. Ann Neurol 2010, 68:295-303.

27. Cossburn M, Pace AA, Jones J, Ali R, Ingram G, Baker K, Hirst C, Zajicek J, Scolding N, Boggild M, Pickersgill T, Ben-Shlomo Y, Coles A, Robertson NP: Autoimmune disease after alemtuzumab treatment for multiple sclerosis in a multicenter cohort. Neurology 2011, 77:573-579.

28. Jones JL, Phuah CL, Cox AL, Thompson SA, Ban M, Shawcross J, Walton A, Sawcer SJ, Compston A, Coles AJ: IL-21 drives secondary autoimmunity in patients with multiple sclerosis, following therapeutic lymphocyte depletion with alemtuzumab (Campath-1H). J Clin Invest 2009, 119:2052-2061.

29. Chen $P$, Lin JJ, Lu CS, Ong CT, Hsieh PF, Yang CC, Tai CT, Wu SL, Lu CH, Hsu YC, Yu HY, Ro LS, Lu CT, Chu CC, Tsai JJ, Su YH, Lan SH, Sung SF, Lin SY, Chuang HP, Huang LC, Chen YJ, Tsai PJ, Liao HT, Lin YH, Chen CH, Chung WH, Hung SI, Wu JY, Chang CF, et al: Carbamazepine-induced toxic effects and HLA-B*1502 screening in Taiwan. N Engl I Med 2011, 364:1126-1133.

30. International Multiple Sclerosis Genetics Consortium; Wellcome Trust Case Control Consortium 2, Sawcer S, Hellenthal G, Pirinen M, Spencer CC, Patsopoulos NA, Moutsianas L, Dilthey A, Su Z, Freeman C, Hunt SE, Edkins S, Gray E, Booth DR, Potter SC, Goris A, Band G, Oturai AB, Strange A, Saarela J, Bellenguez C, Fontaine B, Gillman M, Hemmer B, Gwilliam R, Zipp F, Jayakumar A, Martin R, Leslie $S$, et al: Genetic risk and a primary role for cell-mediated immune mechanisms in multiple sclerosis. Nature 2011, 476:214-219

31. De Jager PL, Chibnik LB, Cui J, Reischl J, Lehr S, Simon KC, Aubin C, Bauer D, Heubach JF, Sandbrink R, Tyblova M, Lelkova P, Steering committee of the BENEFIT study; Steering committee of the BEYOND study; Steering committee of the LTF study; Steering committee of the CCR1 study, Havrdova E, Pohl C, Horakova D, Ascherio A, Hafler DA, Karlson EW: Integration of genetic risk factors into a clinical algorithm for multiple sclerosis susceptibility: a weighted genetic risk score. Lancet Neurol 2009, 8:1111-1119.

32. Oksenberg J, Hauser SL: Genetics of multiple sclerosis. In Multiple Sclerosis. Edited by: Raine CS, McFarland H, Hohlfeld R. London: Saunders Elsevier; 2008:214-225.

33. Comabella $\mathrm{M}$, Vandenbroeck $\mathrm{K}$ : Pharmacogenomics and multiple sclerosis: moving toward individualized medicine. Curr Neurol Neurosci Rep 2011 11:484-491.

34. The Lenercept Multiple Sclerosis Study Group and The University of British Columbia MS/MRI Analysis Group: TNF neutralization in MS: results of a randomized, placebo-controlled multicenter study. Neurology 1999, 53:457-465.

35. Gregory AP, Dendrou CA, Attfield KE, Haghikia A, Xifara DK, Butter F, Poschmann G, Kaur G, Lambert L, Leach OA, Prömel S, Punwani D, Felce JH, Davis SJ, Gold R, Nielsen FC, Siegel RM, Mann M, Bell Il, McVean G, Fugger L: TNF receptor 1 genetic risk mirrors outcome of anti-TNF therapy in multiple sclerosis. Nature 2012, 488:508-511.

\section{Pre-publication history}

The pre-publication history for this paper can be accessed here: http://www.biomedcentral.com/1741-7015/10/116/prepub

doi:10.1186/1741-7015-10-116

Cite this article as: Derfuss: Personalized medicine in multiple sclerosis: hope or reality? BMC Medicine 2012 10:116.

\section{Submit your next manuscript to BioMed Central and take full advantage of:}

- Convenient online submission

- Thorough peer review

- No space constraints or color figure charges

- Immediate publication on acceptance

- Inclusion in PubMed, CAS, Scopus and Google Scholar

- Research which is freely available for redistribution

Submit your manuscript at www.biomedcentral.com/submit 